\title{
NOTE ON DIRICHLET AND FACTORIAL SERIES*
}

BY

\section{TOMLINSON FORT}

Landau $\dagger$ has proved various theorems bearing on the regions of convergence of Dirichlet and factorial series. In the present note I set up a class of series including factorial series and ordinary Dirichlet series, and forming a continuous transition from one to the other. Theorems are proved which include some of Landau's theorems as very special cases.

1. Consider the series

$$
\sum_{n=1}^{\infty} a_{n} A_{n}^{(k)}(z)
$$

where

$$
A_{n}^{(k)}(z)=\frac{k}{z+k-1} \cdot \frac{\Gamma(n k)}{\Gamma(z+n k)} \cdot \frac{\Gamma(z+k)}{\Gamma(k)}
$$

whenever $z, k$ and $n$ have such values that this formula defines a definite number. Whenever, for a particular point $\left(z_{0}, k_{0}, n_{0}\right), A_{n}^{(k)}(z)$ is not defined by the formula but approaches a limit as $(z, k, n)$ approaches $\left(z_{0}, k_{0}, n_{0}\right), A_{n}^{(k)}(z)$ is given at this point the limiting value.

2 . When $k=1$, (1) reduces to the usual factorial series

$$
\sum_{n=1}^{\infty} a_{n} \frac{(n-1) !}{z(z+1) \cdots(z+n-1)} .
$$

3. When $k \rightarrow \infty$, so that $\mid$ am $k \mid \leqq \pi-\epsilon_{1}, \epsilon_{1}>0$, (1) reduces to the ordinary Dirichlet series

$$
\sum_{n=1}^{\infty} \frac{a_{n}}{n^{2}}
$$

To prove this statement consider $A_{n}^{(k)}(z)$ for values of $k$ not equal to zero and so large in absolute value that $z+n k-1 \neq 0(n=1,2,3, \ldots)$ and

* Presented to the Society, December 31, 1919.

tSitzungberichte der mathematisch-physikalischen Klasse der Königlichen Bayerischen Akademie der Wissens c h a f e n z u M ü n c h e n, vol. 36 (1906), pp. 151-218; see especially pp. 167-184. 
$|\operatorname{am}(z+n k-1)| \leqq \pi-\epsilon_{2}, \epsilon_{2}>0$. Then

$$
A_{\cdot n}^{(k)}(z) n^{z}=\frac{k}{z+k-1} \cdot \frac{\Gamma(n k)(n k)^{z}}{\Gamma(z+n k)} \cdot \frac{\Gamma(z+k)}{\Gamma(k) k^{z}} .
$$

Consider the asymptotic form, *

$$
\Gamma(z)=e^{\left(z-\frac{1}{2}\right) \log z-z+\log \sqrt{2 \pi}+\omega(z),}
$$

where $\omega(z)=C_{1} / z+f_{1}(z) / z^{2}, C_{1}$ being a constant and $\left|f_{1}(z)\right|<C_{2}$, a constant, which asymptotic form is valid when $|\mathrm{am} z|<\pi-\epsilon_{3}, \epsilon_{3}>0$. From it one can readily establish the following formula which is fundamental in this paper,

$$
\frac{q^{z} \Gamma(q)}{\Gamma(q+z)}=1+\frac{z-z^{2}}{2 q}+\frac{\psi(z, q)}{q^{2}},
$$

where $|\psi|<M$, a constant, which form is valid over a region where $q \neq 0$, $q+z \neq 0$ and where $|\operatorname{am} q|$ and $|\operatorname{am}(q+z)|$ are both less than $\pi$ mihus some positive constant. From (4) and (5) follows the desired result that when $k \rightarrow \infty$

$$
A_{n}^{(k)}(z) n^{2} \longrightarrow 1
$$

In the remainder of the paper when the symbol $A_{n}^{(k)}(z)$ is used $k$ is in no way restricted to finite values. We shall understand $A_{n}^{(\infty)}(z)=1 / n^{z}$.

4. $\dagger$ If the Dirichlet series (3) converges when $z=z_{0}$, then it converges uniformly over a region defined by the inequality

$$
\left|\operatorname{am}\left(z-z_{0}\right)\right| \leqq \pi / 2-\epsilon_{3}, \epsilon_{3}>0
$$

There exists a straight line $x=x_{0}$, where $z=x+y i$, such that when $x>x_{0}$ (3) converges and when $x<x_{0}$ it diverges. This line is called the "line of convergence."

5. If (1) converges when $z=z_{0}$ and $k=k_{0}$, then it converges uniformly in $z$ and $k$ over any subregion, $R$, of the region defined by the following inequalities

$$
\begin{gathered}
|z|<M,\left|\operatorname{am}\left(z-z_{0}\right)\right| \leqq \pi / 2-\epsilon_{4}, \\
|\operatorname{am} k| \leqq \pi-\epsilon_{5},|k|>\epsilon_{6},
\end{gathered}
$$

$M, \epsilon_{4}, \epsilon_{5}$ and $\epsilon_{6}$ being positive constants, which subregion is so chosen that each of the numbers $|z+k+j|>\epsilon_{7}>0(j=0,1,2, \ldots)$.

* See for example T. H. Gronwall, Annals of Mathematics, vol. 20 (1918-19), pp. 85, 86, 88.

† See for example G. H. Hardy and M. Riesz, The General Theory of Dirichlet Series, p. 3 . 
To prove this theorem we consider $A_{n}^{(k)}(z) n^{z}$ as given by (4). Then by the use of (5) when $n$ is so large, say greater than $n_{0}$, that $|\operatorname{am}(n k+z)|<\pi-\epsilon_{8}$,

$$
\frac{(n k)^{z} \Gamma(n k)}{\Gamma(n k+z)}=1+\frac{z-z^{2}}{2 n k}+\frac{\psi(z, n, k)}{n^{2}}
$$

where $|\psi|<M_{2}$, a constant. Moreover, again by (5), $\Gamma(z+k) /\left(\Gamma(k) k^{z}\right) \rightarrow 1$ as $k \rightarrow \infty$. And since all its finite singularities are excluded from the region in question and $\Gamma$ does not vanish, it remains finite and in absolute value greater than a fixed $\epsilon_{8}>0$. Consequently for $n>n_{0}$,

$$
A_{n}^{(k)}(z) n^{z}=f_{2}(z, k)+\frac{f_{3}(z, k)}{n}+\frac{f_{4}(z, k, n)}{n^{2}},
$$

where $f_{2}, f_{3}$ and $f_{4}$ are analytic in $z$. Moreover we explicitly remark that $\left|f_{2}\right|$, $\left|f_{3}\right|,\left|f_{4}\right|<M$, a constant. It can readily be shown that $1 / A_{n}^{(k)}(z)$ is of the same form and corisequently $A_{n}^{(k)}(z) / A_{n}^{\left(k_{0}\right)}(z)$ also, even if $k_{0} \neq \infty$. For smaller values of $n$, if desired, a convenient definition can be given to $f_{2}, f_{3}$ and $f_{4}$ so that (7) will hold for all values of $n$.

Keep $z$ and $k$ restricted as above and thus assure the validity of (7). Then

$$
\sum_{n=1}^{\infty} a_{n} A_{n}^{(k)}(z)=\sum_{n=1}^{\infty} a_{n} A_{n}^{\left(k_{0}\right)}(z)\left[f_{2}(z, k)+\frac{f_{3}(z, k)}{n}+\frac{f_{1}(z, k, n)}{n^{2}}\right] .
$$

Consequently, ${ }^{*}$ in particular, $\sum_{n=1} a_{n} A_{n}^{(\infty)}(z)$ converges at any point where (1) converges for any other value of $k$ and conversely. Refer to Section 4, and it is immediate that the domain of $z$ includes the half plane to the right of $z_{0}$. Moreover, again by (8) and Section 4, (1) converges uniformly in $z$ and $k$.

6. A common line of convergence of $\sum_{n=1}^{\infty} a_{n} A_{n}^{(k)}(z)$ for all values of $k$ considered follows immediately from Section 5 .

7. Similarly to the results of Section 5 , that is by (8), it is shown that if $\sum_{n=1}^{\infty} a_{n} A_{n}^{\left(k_{0}\right)}(z)$ converges absolutely then so does $\sum_{n=1}^{\infty} a_{n} A_{n}^{(k)}(z), z$ and $k$ restricted to $R$.

8. The finite singularities of $F_{k}(z)=\sum_{n=1}^{\infty} a_{n} A_{n}^{(k)}(z)$ on the line of con-

* Well known results from the theory of infinite series. 
vergence, other than a possible ones caused by a singularity of $\Gamma(z+k)$, are the same and of the same character as those of $D(z)=\sum_{n=1}^{\infty} \frac{a_{n}}{n^{2}}$.

By (4) and (5)

$F_{k}(z)=\frac{k}{2+k-1} \frac{\Gamma(z+k)}{\Gamma(k) k^{2}}\left[D(z)+\frac{z-z^{2}}{k} D(z+1)+\sum_{n=1}^{\infty} \psi(z, k, n) \cdot 1 / n^{2}\right]$. Here $\frac{\Gamma(z+k)}{\Gamma(k) k^{3}}$ and $\sum_{n=1}^{\infty} \psi(z, k, n) \cdot 1 / n^{2}$, under the restrictions imposed on $k$, are analytic in $z$ over any finite region exclusive of the singularities of $\Gamma(z+k)$, while $D(z+1)$ is analytic over a region extending one unit to the left of the line of convergence of the series defining $D(z)$. The theorem follows.

UNIVERSTTY OF ALABAM 4.

UNIVERSTTY, ALA. 